\title{
Diabetes and Renal Disease
}

\author{
Rodica Mihăescu, Corina Şerban, Simona Drăgan, \\ Romulus Timar, Ioana Mozoş, Marius Craina and Adalbert Schiller \\ University of Medicine and Pharmacy „Victor Babeş" Timişoara \\ Romania
}

\section{Introdution}

Diabetes and its devastating complications reduce life expectancy and adversely affect quality of life amongst those affected, thus, posing immense challenges to many societal sectors. The upsurge in the prevalence of diabetes from 171 million in 2000 to 366 million by 2030 as projected by the World Health Organization threatens to overwhelm the economic and healthcare system globally.

In type 1 diabetes the prevalence gradually increases from onset of disease $(6 \%$ after $1-3$ years), reaching over $50 \%$ after 20 years [Warram, 1996]. In type 2 diabetes the prevalence is $20-25 \%$ in both newly diagnosed and established diabetes [Mogensen, 1994]. Approximately $40 \%$ of patients with type 1 diabetes and 20 to $40 \%$ of those with type 2 diabetes will eventually develop diabetic nephropathy, although the reason why not all patients with diabetes develop this complication is unknown.

In both types of diabetes, chronic hyperglycemia is the primary cause of the disease. In type 1 diabetes hyperglycemia starts in the first decades of life and is usually the only recognized cause of nephropathy. On the contrary, in type 2 diabetes hyperglycemia starts after the forties, usually when the kidneys have already suffered the long-term consequences of ageing and of other recognized promoters of chronic renal injury such as arterial hypertension, obesity, dyslipidemia, and smoking [Ruggenenti, 2000]. Similar histological and clinical courses in all diabetic individuals indicated that the pathogenesis of nephropathy is similar in patients with type 1 and type 2 diabetes mellitus.

The kidney is an extremely complex organ with broad ranging functions in the body, including, but not restricted to, waste excretion, ion and water balance, maintenance of blood pressure, glucose homeostasis and generation of erythropoietin or activation of vitamin D. With diabetes, many of these integral processes are interrupted via a combination of hemodynamic and metabolic changes; hyperglycemia also activates a series of changes leading to glomerular and tubular dysfunction and accelerates glomerular cell apoptosis [Forbes, 2010]. Different pathogenetic, hemodynamic and metabolic processes can lead to diabetic kidney disease. In recent years, great progress has been made in understanding the risk factors and pathogenetic processes in diabetic nephropathy. In addition, new insight into the pathophysiology of this chronic disease has lead to better preventive therapies, treatment possibilities and reduced progression to end stage renal disease.

Diabetic kidney disease refers to a characteristic set of structural and functional kidney abnormalities in patients with diabetes. Classic glomerulosclerosis is characterized by 
increased glomerular basement membrane width, diffuse mesangial sclerosis, hyalinosis, microaneurysm, and hyaline arteriosclerosis [Mauer, 1981]. The structural abnormalities include hypertrophy of the kidney, increase in glomerular basement membrane thickness, nodular and diffuse glomerulosclerosis, tubular atrophy, and interstitial fibrosis. The functional alterations include an early increase in glomerular filtration rate with intraglomerular hypertension, subsequent proteinuria, systemic hypertension, and eventual loss of renal function [Ayodele - 2004].

The management of patients with type 2 diabetes and progressive kidney disease requires a comprehensive approach that includes aggressive blood pressure control with agents that also lower urinary protein excretion and optimization of glucose and lipid control, considering the therapeutic limitations imparted by renal dysfunction. Clinicians must also address the comorbidities associated with renal failure such as anemia and secondary hyperparathyroidism. Diabetic nephropathy typically follows a slowly progressive course from albuminuria to azotemia. Consequently, optimal care includes planning for the management of impending renal failure long before the patient requires dialysis or transplantation.

The aim of this chapter is to provide a comprehensive review about the risk factors, the histological changes and the pathogenic mechanisms that lead to diabetic nephropathy/diabetes kidney disease, diagnosis, new biomarkers and recent developments in the prevention and treatment of diabetes kidney disease, targeting the main risk factors and resulting in renal protection and reduced disease progression.

\section{Diabetic nephropathy or diabetic kidney disease?}

The phenomenon of albuminuria has been recognized for more than $200 \mathrm{yr}$, and its association with kidney disease dates to the epochal insights of Richard Bright in 1827 [Cameron, 1988]. The presence of microalbuminuria is associated with increased cardiovascular morbidity and mortality, and regular screening is recommended in guidelines for diabetes care. Albuminuria reflects functional and potentially reversible abnormalities initiated by glomerular hyperfiltration, proteinuria, a size-selective dysfunction of the glomerular barrier normally associated with glomerular filtration rate (GFR) decline that may result in end-stage renal disease [Ruggenenti, 2006].

The Diabetes and Chronic Kidney Disease work group of the National Kidney Foundation Kidney Disease Outcomes Quality Initiative suggested that chronic kidney disease considered to be caused by diabetes should be named "diabetic kidney disease" and the term "diabetic nephropathy" should be reserved for kidney disease attributed to diabetes with histopathological changes, demonstrated by renal biopsy [Cavanaugh - 2007]. Progression from normoalbuminuria to microalbuminuria defines the initiation of diabetic nephropathy, and the transition from microalbuminuria to overt diabetic nephropathy resulting in deterioration of renal function and end-stage renal disease constitutes its progression.

Diabetic kidney disease is characterized by persistent albuminuria $(>300 \mathrm{mg} / \mathrm{dl}$ or $>200$ $\mu \mathrm{g} / \mathrm{min}$ ) that is confirmed on at least 2 occasions 3 to 6 months apart, with a progressive decline in the glomerular filtration rate (GFR), elevated arterial blood pressure, and an increased risk for cardiovascular morbidity and mortality.

\section{The initiation of diabetic kidney disease}

Generalized endothelial dysfunction and inflammation are important antecedents of microalbuminuria in both types of diabetes. Markers of endothelial dysfunction, including 
elevated serum von Willebrand factor (vWF) and increased transcapillary albumin escape rate, are present before the onset of microalbuminuria in type 1 diabetes and worsen in association with it. Type 2 diabetes is often complicated by the presence of other risk factors for vascular disease, and discerning the contribution of hyperglycemia and its sequel to endothelial dysfunction in this condition is more difficult [Satchell, 2008]. While in some type 2 diabetic patients microalbuminuria may occur in the absence of evidence of endothelial dysfunction, in others, vWF levels predict its development [Stehouwer, 2002]. Markers of chronic low-grade inflammation, including C-reactive protein (CRP), are also correlated with microalbuminuria in type 1 and type 2 diabetes [Schalkwijk, 1999].

\section{The natural progression of diabetic kidney disease}

In an initial phase, diabetic kidney disease is characterized by glomerular hyperfiltration due to a reduction in the resistance of the afferent and efferent glomerular arterioles, and consequent increased renal perfusion. The natural progression of diabetic nephropathy has been well studied in type 1 diabetes by Mogenson et al. There are 5 stages of diabetic kidney disease:

Stage 1: Stage of hyperfiltration is characterized by renal hypertrophy and renal hyperfiltration. Increased glomerular filtration leads to increased urinary albumin excretion rate (increases by 20- 40 percent) along with increase in renal blood flow by $9-14$ percent.

Stage 2: Renal hyperfiltration with increased glomerular filtration pressure persists, but there is normalization of urinary albumin excretion rate and normal blood pressure.

Stage 3: Incipient nephropathy or stage of microalbuminuria is characterised by urinary albumin excretion rate of 30 -300 mg per day, with a rise of blood pressure. More characteristic is the impaired nocturnal dip in blood pressure. Microscopically, the renal biopsy (or histology) shows increased basement membrane thickness and increased mesangium.

Stage 4: Overt nephropathy is characterized by progressive drop in glomerular filtration rate by $10 \mathrm{ml}$ per minute per year, while proteinuria increases at the rate of $15-40$ percent per year and hypertension ensues.

Stage 5: End stage renal disease occurs at about $7-14$ years from stage 4 requiring renal replacement therapy.

However, it is often difficult to document these various stages in a diabetic patient in clinical practice because of confounding factors, such as blood pressure medications, which modify the natural course of diabetic nephropathy.

A greater proportion of patients with type-2 diabetes compared with type-I diabetes have microalbuminuria and overt nephropathy at or shortly after diagnosis of diabetes. This is because the disease may have been present for several years before the diagnosis. In addition, concomitant presence of hypertension at the time of diagnosis also contributes to the high prevalence of microalbuminuria in type-2 diabetes. Progression from microalbuminuria to overt nephropathy occurs in $20-40 \%$ of Caucasians within a 10 -year period, with approximately $20 \%$ of those with overt nephropathy progressing to ESRD over a period of 20 years [Ayodele, 2004].

\section{Epidemiology}

Diabetic kidney disease is one of the most serious complications of diabetes mellitus and is the leading cause of end stage renal disease both in the USA and in Europe, requiring renal 
replacement therapy. As the population of patients with diabetes of long duration grows, reports of a dramatically increasing burden of diabetic nephropathy are appearing. Globally the burden of diabetes is expected to double between 2000 and 2030, with the greatest increases in prevalence expected to occur in the Middle East, sub-Saharan Africa, and India (KDOQI Clinical Practice Guidelines). This increased risk and more rapid progression of diabetic nephropathy also has been reported in immigrants from developing to developed countries (KDOQI Clinical Practice Guidelines) [Burney, 2009].

Diabetic kidney disease affects males and females equally and rarely develops before 10 years' duration of type $1 \mathrm{DM}$. The peak incidence (3\%/year) is usually found in persons who have had diabetes for 10-20 years. The mean age of patients who reach end-stage kidney disease is about 60 years. Although in general, the incidence of diabetic nephropathy is higher among elderly persons who have had type 2 diabetes for a longer period, the role of age in the development of diabetic kidney disease is unclear. In Pima Indians with type 2 diabetes, the onset of diabetes at a younger age was associated with a higher risk of progression to end-stage kidney disease.

\section{Risk factors}

Although diabetes is often accompanied by hyperglycemia, hypertension, and altered lipid profile, surprisingly, most individuals with type 1 or type 2 diabetes do not develop diabetic nephropathy. This suggests that other factors are involved [Hall, 2006]. Persuasive evidences have implicated also nontraditional risk factors including lipid disorders, chronic inflammation and anemia in the development of diabetic kidney disease.

\subsection{Race}

The incidence and severity of diabetic kidney disease are increased in blacks, Mexican Americans, Pima Indians, and Hispanics compared with Caucasians. Even after adjusting for confounding factors such as lower socioeconomic status and increased incidence of hypertension in blacks, there is still a 4.8 times greater risk of ESRD in blacks compared to Caucasians [Ayodele, 2004].

\subsection{Aging}

Ageing is per se a cause of progressive glomerulosclerosis and combined with the risk factors may contribute to the specific arteriolosclerotic changes, that often coexist with, and occasionally overwhelm, the typical features of diabetic glomerulopathy, in particular in type 2 diabetes [Ruggeneti, 1998]. Renal blood flow and GFR diminish over time in elderly persons, minimized by a rise in the filtration fraction. In older diabetic patients, the decrease in kidney mass, particularly the renal cortex, and the histological changes of diabetic nephropathy are compounded by advanced vascular changes. Pathologically, the aging kidney may be associated with basement membrane thickening and mesangial expansion that are also key histological features of diabetic glomerulopathy. Global glomerulosclerosis affecting the kidneys of elderly persons may relate to hyperperfusion, also observed in diabetes. However, studies about the diagnosis and prevalence of diabetic kidney disease in the elderly are lacking [Williams, 2009].

\subsection{Smoking}

Smoking is a risk factor for diabetic nephropathy and might contribute to its progression [64]. Although some studies did not confirm these observations, it is strongly recommended 
to quit smoking in any phase of $\mathrm{DN}$, also aiming to reduce the associated cardiovascular and cancer risk [Luk, 2008].

\subsection{Genetic risk factors}

Genetic susceptibility is one of the determinants of progression and severity in diabetic kidney disease. Several genes predisposing to type 2 diabetes have recently been identified. In addition to the recognized and powerful effects of environmental factors, there is abundant evidence in support of genetic susceptibility to the microvascular complication of nephropathy in individuals with both type 1 and type 2 diabetes. Familial aggregation of phenotypes such as end-stage renal disease, albuminuria, and chronic kidney disease have routinely been reported in populations throughout the world, and heritability estimates for albuminuria and glomerular filtration rate demonstrate strong contributions of inherited factors. Recent genome-wide linkage scans have identified several chromosomal regions that likely contain diabetic nephropathy susceptibility genes, and association analyses have evaluated positional candidate genes under these linkage peaks. These complimentary approaches have demonstrated that polymorphisms in the carnosinase 1 gene on chromosome 18q, the adiponectin gene on $3 q$, and the engulfment and cell motility gene on $7 p$ are likely associated with susceptibility to diabetic nephropathy [Freedman - 2007].

\subsection{Arterial hypertension}

Increase of arterial blood pressure is an early and frequent phenomenon in diabetic nephropathy as well as in the development of macrovascular lesions. There has been some recent evidence that genetic predisposition to hypertension may predispose to the development of diabetic kidney disease. In diabetic kidney disease hypertension is not merely the result of relentless kidney damage. There is considerable clinical evidence that the elevated arterial pressure is also important in the genesis of the glomerular lesion. Indeed the development of proteinuria is paralleled in most cases by a gradual increase in systemic blood pressure, and there is a significant correlation between the blood pressure levels and the rate of decline in glomerular filtration rate [Mogensen, 1985]. In type 2 diabetes, lowering blood pressure, regardless of the used agent, retards the onset and progression of diabetic nephropathy [Schrier, 2002]. In type 1 diabetes, Lurbe et al. have noted that an insufficient decline in nighttime blood pressure (nondipping) preceded the onset of microalbuminuria [Lurbe, 2002]. Although the type 2 diabetic patient is usually hypertensive before the onset of nephropathy, nephropathy aggravates the severity of hypertension.

\subsection{Hyperglycemia}

The hyperglycemic state itself is a strong risk factor for diabetic kidney disease and causes the proliferation of mesangial cells and their matrix, as well as the thickening of the basement membrane. In recent years, many discoveries elucidated the mechanisms by which hyperglycemia affect the renal glomerular and tubulointerstitial cells and suggested that podocyte injury have a pivotal role in the pathogenesis of diabetic glomerulopathy. Molecular pathogenesis of diabetic podocyte injury is likely multifactorial involving a number of interrelated signaling pathways that have yet to be well understood. Sustained hyperglycemia affects the glomerular cells by various mechanisms. The changes lead to altered structure and function in the glomerulus. 


\subsection{Lipid disorders}

Patients with diabetes have a variety of disorders of plasma lipids. These lipid abnormalities are known to contribute to cardiovascular risk. The role of lipids in diabetic nephropathy is not clear [Hall, 2006]. In type 2 DM, elevated serum cholesterol is a risk factor for the development of DN. In type $1 \mathrm{DM}$ patients increased serum triglycerides, total and LDLcholesterol were associated with micro- and macroalbuminuria. High serum cholesterol also seems to be a risk factor for GFR loss in macroalbuminuric type 1 diabetic subjects [ Zelmanovitz - 2009].

For many decades, obesity has been known to cause structural changes to the renal parenchyma, and biopsies from obese subjects consistently showed glomerulomegaly with or without focal segmental glomerulosclerosis. Several mechanisms have been proposed to explain obesity-related renal changes. One explanation is the greater work load imposed on the kidneys as a direct result of larger body mass, as increased tissue turnover and toxic output place additional strains on the nephrons. Another hypothesis is lipotoxicity: the exposure of renal tissues to excess free fatty acid leads to generation of oxidative stress and cytotoxic lipid products. Recent attention has focused on adipose tissue as a rich source of pro-inflammatory cytokines and growth factors such as tumor necrosis factor a, interleukin6 and leptin. The plethora of circulating cytokines is believed to have direct effects on renal hemodynamics and glomerular cellularity [Luk, 2008].

Ravid et al. found that the level of cholesterol both at onset and after a five-year follow-up period was positively related with the subsequent increase in urinary albumin excretion in microalbuminuric patients with type-2 diabetes [Ravid, 1995]. Gall et al. found that total serum cholesterol was significantly associated with development of abnormally increased urinary albumin excretion [Gall, 1997]. Klein et al. in a study of type-I individuals, found that higher total serum cholesterol and lower HDL cholesterol were associated with incidence of renal insufficiency [Klein, 1999].

\subsection{Increased protein intake}

Protein intake: 0.8 - 1.0 gram per kilogram body weight per day in patients with normal renal functions and decrease to $0.6-0.8$ gram per kilogram body weight per day once proteinuria sets in. High intakes of animal protein have been shown to increase renal blood flow and glomerular filtration rate (GFR) whereas soy protein does not appear to have this effect. Substituting soy protein for animal protein has been found to decrease hyperfiltration in subjects with diabetes and may reduce urine albumin excretion [Song, 2004].

\subsection{Chronic inflammation}

With the recognition of the central role played by inflammatory processes in cardiovascular disease and both type 1 and type 2 diabetes, the association between potentially modifiable inflammatory biomarkers and clinical outcomes such as nephropathy and cardiovascular disease in diabetes is of interest. Moreover, although diabetic nephropathy has traditionally not been considered an inflammatory nephritis, more recent evidence that macrophage accumulation is characteristic for diabetic glomerulosclerosis has challenged this perception [Foruta, 1993].

Recent studies suggested that inflammatory processes and immune cells might be involved in the development and progression of diabetic nephropathy. Infiltrated macrophages are found within renal diabetic tissues, and recent studies demonstrated that macrophagederived products can induce further inflammation in the diabetic kidney. Furthermore, 
activated $\mathrm{T}$ lymphocytes have been associated with diabetic nephropathy. One of the most striking features of leukocytes from patients with diabetes is the activated status of blood neutrophils. There is no doubt that immune cells participate in the vascular injury in the conditions of diabetic nephropathy, and their migration into the kidney is a crucial step in the progression of this disease [Galkina - 2006]. A few cross-sectional studies of individuals with type 1 diabetes have reported associations between increased albumin excretion rate and elevated levels of inflammatory markers including high-sensitivity (hs) C-reactive protein (CRP), soluble (s) tumor necrosis factor- $\alpha$ receptor-1 (TNFR-1), intercellular adhesion molecule-1 (ICAM-1), and vascular cell adhesion molecule-1 (VCAM-1), but whether the elevation of these inflammatory markers precedes nephropathy progression is not well described.

Inflammatory cytokines and growth factors, mainly VEGF, TGF-1, IL-1, IL-6, and IL-18, as well as TNF-a, are also involved in the development and progression of DKD [NavarroGonzales, 2008]. If inflammation contributes to the development of nephropathy, it would represent a potential therapeutic target for slowing the microvascular and macrovascular complications of diabetes [Lin, 2008].

\subsection{Anemia}

Anemia is a common finding in patients with diabetes, particularly in those with overt nephropathy or renal impairment. Anemia in patients with diabetes is not restricted to those with renal impairment, as about half have normal renal function. Failure to up-regulate erythropoietin production in response to a declining hemoglobin level has been suggested to be the primary mechanism leading to chronic anemia. Accordingly, the presence of anemia may be a distinctive marker for renal tubulointerstitial dysfunction even before overt nephropathy manifests [Luk, 2008]. The precise mechanisms by which diabetes impairs the renal erythropoietin response to reduced $\mathrm{Hb}$ remain to be established. While functional erythropoietin deficiency is clearly linked to renal dysfunction in diabetes, the reduction in synthesis of erythropoietin in response to anemia appears to be beyond that seen in other renal (and particularly glomerular) diseases. A number of mechanisms potentially contribute to the preferential development of anemia in patients with diabetes. For example, the predominance of damage to specific cells and to the vascular architecture of the renal tubulo-interstitium, and the resulting systemic inflammation, autonomic neuropathy, and induction of inhibitors of erythropoietin release, have all been suggested as contributors to impaired renal erythropoietin production and release [Thomas, 2006]. Although anemia can be considered a marker of kidney damage, reduced hemoglobin levels independently identify diabetic patients with an increased risk of microvascular complications, cardiovascular disease and mortality. Nevertheless, a direct role in the development or progression of diabetic complications remains to be clearly established and the clinical utility of correcting anemia in diabetic patients has yet to be demonstrated in randomized controlled trials. Correction of anemia certainly improves performance and quality of life in diabetic patients [Thomas, 2007].

\subsection{Hyperuricemia}

Uric acid has been associated with renal disease, even though hyperuricemia may be a marker of or by itself be responsible for microvascular disease in diabetes. In animal models, elevated level of uric acid can lead to arteriolopathy of preglomerular vessels, impaired 
autoregulation, glomerular hypertension, as well as endothelial dysfunction. Kidney damage in hyperuricemic rats is not dependent on blood pressure, and instead involves the renin-angiotensin system. In patients with diabetes, serum uric acid early in the course of diabetes is significantly, and independent of confounders, associated with later development of persistent macroalbuminuria. Therefore, uric acid may be a novel and important player in the pathogenesis of microvascular complications in diabetes. A doseresponse relationship between serum uric acid and early decline in renal function has recently been demonstrated in patients with type-1 diabetes [Hovind, 2011].

\subsection{Aldosterone}

Another deleterious factor that has been underappreciated until recently is aldosterone. In addition to the classic effects of promoting sodium retention and volume expansion, aldosterone promotes inflammation, production of cytokines, growth factors and endothelial dysfunction, which acts on the renal vasculature, glomeruli and tubules and favors the emergence of proteinuria. In the setting of diabetes and longstanding hyperglycemia, free amino groups of proteins are non-enzymatically modified by glucose and its metabolites to form Schiff bases that eventually lead to the formation of advanced glycation end products (AGEs).

\subsection{The use of oral contraceptives}

Oral contraceptive (OC) use is associated with increased intrarenal renin-angiotensinaldosterone system (RAA System) activity and risk of nephropathy, though the contribution of progestins contained in the OC in the regulation of angiotensin-dependent control of the renal circulation has not been elucidated [Sarna, 2009].

\section{Pathogenic mechanisms}

Multiple mechanisms contribute to the development and outcomes of diabetic nephropathy, such as an interaction between hyperglycemia induced metabolic and hemodynamic changes and genetic predisposition, which sets the stage for kidney injury [Dronavalli 2008]. Extracellular matrix accumulation is one of the hallmarks in the development of the disease that leads to the formation of glomerular and interstitial lesions [Deckert, 1981]. Multiple biochemical pathways have been postulated that explain how hyperglycemia causes tissue damage: nonenzymatic glycosylation that generates advanced glycosylation end products, activation of protein kinase $\mathrm{C}$, and acceleration of the polyol pathway.

\section{Increased advanced glycation end products (AGEs)}

Advanced glycation end products (AGEs) are diverse group of molecules and are well known heterogeneous compounds formed nonenzymatically through an interaction of reducing sugar with free amino group of proteins, lipids and nucleic acids [Krishan, 2010]. AGEs form at a constant rate in the normal body; however, in diabetes, this process is drastically increased. Accumulation of AGEs in the kidney may contribute to the progressive alteration in renal architecture and loss of renal function in patients and rodents via various mechanisms, including their cross-linking properties of matrix proteins and activation of the downstream signaling. AGEs may produce functional changes in the kidney by cross-linking with the glomerular basement membrane and other vascular 
membranes. AGE binding proteins may also be involved. The best defined is the receptor for AGEs (RAGE). Three main consequences have been found in association with AGEs inside cells: (1) functional alterations of intracellular proteins, (2) altered interaction with AGE receptors, and (3) altered interactions with matrix and other cells. Binding of AGEs to RAGEs activates cell signaling mechanisms coupled to increased transforming growth factor- $\$$ (TGF- $\$$ ) and vascular endothelial growth factor (VEGF) expression, which are increased in diabetic nephropathy and are thought to contribute to diabetes complications.

\subsection{Increased transforming growth factor $ß 1$}

Studies in rodent models have suggested that reduction in renal transforming growth factor (TGF)- $\beta 1$ may underlie the renoprotective effects of the renin-angiotensin system (RAS) blockade [Langham, 2006]. Over the past several years, experimental evidence consistently has suggested a key role for TGF- $\beta$ in the pathogenesis of the extracellular matrix accumulation that characterizes diabetic nephropathy and closely correlates with declining renal function [Mauer, 1984]. Angiotensin II potently induces synthesis of transforming growth factor (TGF)- $\beta$, a profibrotic growth factor consistently implicated in the pathogenesis of diabetic nephropathy [Border, 1996]. The mechanisms for this fibrogenic or prosclerotic action of TGF- $\beta$ are multiple and include both stimulation of extracellular matrix synthesis and inhibition of its degradation [Bruijn, 1996].

\subsection{Activation of protein kinase $C ß$ (PKCß)}

Other proposed mechanisms by which hyperglycemia promotes the development of diabetic nephropathy include activation of PKC that involves de novo formation of diacylglycerol and oxidative stress. Hyperglycemia also increases the generation of advanced products of nonenzymatic glycosilation of proteins through activation of aldol reductase pathway and protein kinase $\mathrm{C}$ (PKC). The final products of non-enzymatic glycosilation are bound to collagen and proteins that constitute the glomerular basement membrane and make the glomerular barrier more permeable to the passage of proteins, resulting in increased urinary albumin excretion [Zelmanovitz, 2009]. Two clinical observations explain the possible mechanisms involved. The first is that the hyperglycemic state seems to sensitize the endothelium to injury from elevated blood pressure. The second observation is that successful pancreas transplant that results in normal insulin regulation and normoglycemia is associated with a reversal of the lesions of diabetic nephropathy [Lurbe, 2002]. Specifically, activation of this enzyme leads to increased secretion of vasodilatory prostanoids, which contributes to glomerular hyperfiltration. By activation of TGF- $\beta 1$, PKC might also increase production of extracellular matrix by mesangial cells. PKC activation induces the activity of mitogen-activated protein kinases (MAPK) in response to extracellular stimuli through dual phosphorylation at conserved threonine and tyrosine residues. The coactivation of PKC and MAPK in the presence of high glucose concentrations indicates that these two families of enzymes are linked [Dronavalli, 2008].

\subsection{Aldose reductase pathway/polyol pathway}

Aldose reductase (AR) is a monomeric reduced nicotinamide adenine dinucleotide (NAD) phosphate (NADPH)-dependent enzyme and a member of aldo-keto reductase superfamily. Aldose reductase (AR) is widely expressed aldehyde-metabolizing enzyme. The polyol pathway is implicated in the pathogenesis of diabetic nephropathy. A number of studies 
have shown a decrease in urinary albumin excretion in animals administered aldose reductase inhibitors, but in humans these agents have not been studied widely and the results are inconclusive [Tilton, 1989]. The reduction of glucose by the AR-catalyzed polyol pathway has been linked to the development of secondary diabetic complications [Srivastava - 2005].

\subsection{The protein kinase C (PKC) pathway}

The protein kinase $\mathrm{C}(\mathrm{PKC})$ superfamily comprises proteins that are activated in response to various pathogenic stimuli in the diabetic state. Hyperglycemia is the predominant stimulus that induces the activation of distinct PKC isoforms within a cell, each mediating specific functions, probably through differential subcellular localization. Craven and DeRubertis [Craven, 1989] and Lee et al. [Lee, 1989] were the first to provide data indicating that activation of the PKC system by hyperglycemia may represent an important pathway by which glucotoxicity is transduced in susceptible cells in diabetic nephropathy. The putative intracellular mechanism is the glucose induced de novo synthesis of diacylglycerol that is one of the intracellular activators of PKC [Menne, 2009]. PKC activation is involved in the regulation of vascular permeability and contractility, endothelial cell activation and vasoconstriction, extracellular matrix (ECM) synthesis and turnover, abnormal angiogenesis, excessive apoptosis, leukocyte adhesion, abnormal growth factor signaling and cytokine action, as well as abnormal cell growth and angiogenesis, all of which are involved in the pathophysiology of diabetic vascular complications [Meier, 2009].

\subsection{Vascular endothelial growth factor (VEGF) pathway}

Vascular endothelial growth factor (VEGF) is a key regulator of vascular permeability and angiogenesis and is implicated in the pathogenesis of diabetic retinal neovascularisation. In the glomerulus it is produced in large amounts by podocytes and is thought to be important in maintaining glomerular endothelial cell fenestrations [Satchell, 2006]. Hyperglycemia increases the expression of vascular endothelial growth factor (VEGF) in podocytes causing increased vascular permeability. VEGF has the potential to induce the new vessel growth seen in early diabetic nephropathy and to alter the permeability characteristics of the endothelium [Kanesaki, 2005]. In human type 1 diabetes, serum VEGF concentrations vary according to glycemic control, and higher levels are associated with microvascular complications, including microalbuminuria [Chiarelli, 2000]. In type 2 diabetes, VEGF is upregulated early in the course of disease and urinary VEGF levels are correlated with microalbuminuria [Kim, 2005]. Further studies have confirmed initial upregulation of VEGF signalling in type 2 diabetes followed by a down-regulation with podocyte loss and sclerosis development [Hohenstein, 2006].

\subsection{Oxidative stress pathway}

A large body of evidence indicates that oxidative stress is the common denominator link for the major pathways involved in the development and progression of diabetic micro- as well as macrovascular complications of diabetes nephropathy. Both hyperglycemia and activation of the renin-angiotensin system play a role in the generation of reactive oxygen species (ROS) [Anjaneyulu, 2004]. There are a number of macromolecules that have been implicated for increased generation of reactive oxygen species (ROS), such as, NAD(P)H oxidase, advanced glycation end products (AGE), defects in polyol pathway, uncoupled 
nitric oxide synthase (NOS) and mitochondrial respiratory chain via oxidative phosphorylation. Excess amounts of ROS modulate activation of protein kinase C, mitogenactivated protein kinases, and various cytokines and transcription factors which eventually cause increased expression of extracellular matrix (ECM) genes with progression to fibrosis and end stage renal disease. Activation of renin-angiotensin system (RAS) further worsens the renal injury induced by ROS in diabetic nephropathy. Buffering the generation of ROS may represent a promising therapeutic to ameliorate renal damage from diabetic kidney disease; however, various studies have demonstrated minimal reno-protection by these agents [Kashihara - 2010]. These derangements, along with hemodynamic changes, activate various cytokines and growth factors such as vascular endothelial growth factor, transforming growth factor- $\beta$, Interleukin 1 (IL 1), IL-6 and IL-18.

\subsection{The activation of the transcription factor NF-kB}

Patients with diabetic kidney disease have higher monocyte NFKB activity than diabetic patients without renal complications. The transcription factor NF-kB helps to control the expression of numerous genes activated during inflammation. NF-kB is induced by various cell stress-associated stimuli including growth factors, vasoactive agents, cytokines, and oxidative stress. NF-kB in turn controls the regulation of genes encoding proteins involved in immune and inflammatory responses (i.e., cytokines, chemokines, growth factors, immune receptors, cellular ligands, and adhesion molecules) [Schmid - 2006]. Inhibition of NFKB reduces interstitial monocyte infiltration in rats with proteinuria, and NFKB is believed to play a key role in proteinuria-induced tubulointerstitial damage in diabetes [Remuzzi, 1998].

\section{The histological changes}

Three major histological changes occur in the glomeruli of persons with diabetic kidney disease. First, mesangial expansion is directly induced by hyperglycemia, perhaps via increased matrix production or glycosylation of matrix proteins. Second, thickening of the glomerular basement membrane occurs. Third, glomerular sclerosis is caused by intraglomerular hypertension (induced by dilatation of the afferent renal artery or from ischemic injury induced by hyaline narrowing of the vessels supplying the glomeruli). These different histological patterns appear to have similar prognostic significance. All of these are part and parcel of microvascular complications of diabetes. A large body of evidence indicates that oxidative stress is the common denominator link for the major pathways involved in the development and progression of diabetic micro- as well as macro-vascular complications of diabetes.

Light microscopy findings show an increase in the solid spaces of the tuft, most frequently observed as coarse branching of solid (positive periodic-acid Schiff reaction) material (diffuse diabetic glomerulopathy). Large cellular accumulations also may be observed within these areas. These are circular on section and are known as the Kimmelstiel-Wilson lesions/nodules.

Electron microscopy provides a more detailed definition of the structures involved. In advanced disease, the mesangial regions occupy a large proportion of the tuft, with prominent matrix content. Further, the basement membrane in the capillary walls (ie, the peripheral basement membrane) is thicker than normal. Immunofluorescence microscopy may reveal deposition of albumin, immunoglobulins, fibrin, and other plasma proteins 
along the GBM in a linear pattern most likely as a result of exudation from the blood vessels, but this is not immunopathogenetic or diagnostic and does not imply an immunologic pathophysiology. The renal vasculature typically displays evidence of atherosclerosis, usually due to concomitant hyperlipidemia and hypertensive arteriosclerosis.

The severity of diabetic glomerulopathy is estimated by the thickness of the peripheral basement membrane and mesangium and matrix expressed as a fraction of appropriate spaces (eg, volume fraction of mesangium/glomerulus, matrix/mesangium, or matrix/glomerulus). The glomeruli and kidneys are typically normal or increased in size initially, thus distinguishing diabetic nephropathy from most other forms of chronic renal insufficiency, wherein renal size is reduced (except renal amyloidosis and polycystic kidney disease).

\section{Clinical diagnosis of diabetic kidney disease}

The clinical diagnosis of diabetic kidney disease is, mainly, based on detection of albuminuria from spot urine is recommended because the albumin-creatinine ratio by spot urine sample has demonstrated excellent correlation with the 24-hour urine albumin measurements. Estimated glomerular filtration rate (eGFR), has been added to the albuminuria also as a renal biomarker. Both are indeed associated with renal and cardiovascular disease in individuals with diabetes and may be used to identify individuals at risk of long-term complications.

Microalbuminuria, which is an indicator of endothelial dysfunction, and an independent marker for cardiovascular morbidity and mortality in individuals with and without diabetes is defined as an albumin-creatinine ratio of $30-300 \mathrm{mg} / \mathrm{g}$ from a spot urine collection, urinary albumin excretion $30-300 \mathrm{mg} / 24$ hours in a 24 -hour urine collection, or $20-200$ $\mu \mathrm{g} / \mathrm{min}$ in a timed urine collection.

Macroalbuminuria is defined as an albumin/creatinine ratio (ACR) $>300 \mathrm{mg} / \mathrm{g}$ from a spot urine collection, urinary albumin excretion $>300 \mathrm{mg} / 24$ hours in a 24-hour urine collection, or $>200 \mu \mathrm{g} / \mathrm{min}$ in a timed urine collection. For initial screening of diabetic kidney disease, the measurement of albuminuria from spot urine is recommended because the ACR by spot urine sample has demonstrated excellent correlation with the 24-hour urine albumin measurements.

If the determination of albuminuria cannot be performed at diagnosis, we can measure proteinuria. Microproteinuria is defined by a value between $300-500 \mathrm{mg} / 24$ hours and macroproteinuria is defined by a value higher than $500 \mathrm{mg} / 24$ hours. Serum markers of glomerular filtration rate and microalbuminuria identify renal impairment in different segments of the diabetic population, indicating that serum markers as well as microalbuminuria tests should be used in screening for nephropathy in diabetic patients.

There is accumulating evidence suggesting that the risk for developing diabetic nephropathy and cardiovascular disease starts when UAE values are still within the normoalbuminuric range [Gerstein, 2001]. After the diagnosis of micro- or macroalbuminuria is confirmed, patients should undergo a complete evaluation, including a work-up for other etiologies and an assessment of renal function and the presence of other comorbid associations [Gross - 2005].

\subsection{Glomerular filtration rate}

Although the best measure for glomerular filtration rate (GFR) is obtained by techniques that involve infusion of exogenous substances, GFR is usually estimated in clinical practice 
by various formulae based on serum creatinine concentration, since this is much less invasive and time-consuming. The most popular equation used today is the Modification of Diet in Renal Disease (MDRD) equation [Levey, 1999 ].

Recent efforts to identify novel biomarkers in the diabetic population have been evolving either to detect early kidney injury or repair that ultimately lead to progression of diabetic kidney disease.

\subsection{Cystatin-C}

Cystatin-C, a cysteine protease inhibitor that is produced by virtually all nucleated cells and released into the bloodstream is entirely filtered by the kidney glomerulus and metabolized by the proximal tubule. Recently interest has developed in cystatin- $\mathrm{C}$ and was proposed as a potential endogenous filtration marker of GFR because it is a more sensitive indicator of mild renal impairment and may better estimate the GFR than serum creatinine.

\subsection{Retinol-binding protein 4 (RBP-4)}

Retinol-binding protein 4 (RBP-4) is a small visceral protein, mainly synthesized in the liver and catabolized in the kidneys after glomerular filtration. Initially reported as an adipokine that impairs insulin sensitivity, the concentrations of this adipokine are increased in diabetic patients as compared to normal subjects.

Mechanistic studies have suggested that RBP-4 impaired insulin sensitivity by inhibition of insulin receptor substrate-1 phosphorylation and phosphatidylinositol 3-kinase activation in muscle and by induction of glucose production in liver via cytosolic phosphoenolpyruvate carboxykinase (PEPCK) stimulation [Yang, 2005]. Interestingly, urinary RBP-4 excretion is increased in early diabetic nephropathy and might even be a marker of early renal damage preceding microalbuminuria [Hong, 2000].

\subsection{Connective tissue growth factor (CTGF)}

Connective tissue growth factor (CTGF) is a polypeptide with functions in extracellular matrix production and other profibrotic activity mediated by transforming growth factor- $\beta 1$. Other biological functions of CTGF include angiogenesis, chondrogenesis, osteogenesis, and cell adhesion, migration, proliferation, and differentiation. The upregulation of CTGF has been observed in human and experimental diabetic nephropathy.

\subsection{Pigment epithelium-derived factor (PEDF)}

Pigment epithelium-derived factor (PEDF) is a glycoprotein that belongs to the superfamily of serine protease inhibitors with complex neurotrophic, neuroprotective, anti-angiogenic, anti-oxidative and anti-inflammatory properties, any of which could potentially be exploited as a therapeutic option for the treatment of vascular complications in diabetes. Recent studies suggest that PEDF could reduce proteinuria by suppressing podocyte damage and decreased nephrin as well as increased VEGF expression in the glomeruli of ADN rats. It has been reported that PEDF counteracts the effects of VEGF [Liu, 2004] and advanced glycation end products [Inagaki, 2003]. It is noteworthy in the future to determine whether PEDF may be used as a specific marker for screening incipient DN.

\subsection{Pentoxifylline (PTF)}

Pentoxifylline (PTF) is a methylxanthine derivative with favorable effects on microcirculatory blood flow as a result of its rheological properties. Recent studies have shown that PTF 
reduces urinary protein excretion in individuals with diabetes, both with normal renal function and with renal insufficiency. In a previous study in patients with diabetes and nephropathy, was found that TNF-a concentrations were significantly elevated and related to the urinary protein excretion and, moreover, that the levels of this cytokine decreased after PTF administration, with a significant correlation between the decrease of TNF- $\alpha$ and the reduction of proteinuria. It was demonstrated in rats that Pentoxifylline reduces the accumulation and proliferation of glomerular macrophages in mesangial proliferative glomerulonephritis, and the recruitment of macrophages, lymphocytes, and major histocompatibility complex (MHC) class II antigen-positive cells into remnant kidney interstitium [Lin, 2002].

Emerging roles of urinary biomarkers have proven beneficial due to the ability to standardize the various markers to creatinine or peptides already present in the urine. The role of neutrophil gelatinase-associated lipoprotein, kidney injury molecule- 1 and podocin derivatives have gained enormous significance in the process of early identification of kidney injury in the diabetic population [Buddineni - 2011].

\section{Differential diagnosis}

Differential diagnosis is usually based on the history, physical examination, laboratory evaluation, and imaging of the kidneys. Renal biopsy is only recommended in special situations. The diagnosis of diabetic nephropathy is easily established in long-term type 1 diabetic patients ( $>10$ years diabetes duration), especially if retinopathy is also present. Typical diabetic nephropathy is also likely to be present in proteinuric type 2 diabetic patients with retinopathy. However, diagnostic uncertainty exists in some patients with type 2 diabetes since the onset of diabetes is unknown and retinopathy is absent in a significant proportion (28\%) of these patients [Christensen, 2001].

Imaging of the kidneys, usually by ultrasonography, should be performed in patients with symptoms of urinary tract obstruction, infection, or kidney stones or with a family history of polycystic kidney disease [Levey, 2003].

\section{Prevention and treatment of diabetic nephropathy}

Monitoring of type 2 diabetic patients for microalbuminuria at least once per year is recommended. Microalbuminuria in type 2 diabetic patients is not as potent a real risk predictor as in type 1 diabetic patients, but it is certainly the best available tool to identify patients in need of intensified treatment [Rugenetti, 1998].

\section{Glycemic and blood pressure control}

The interventions which proved to prevent the onset or attenuate the progression of diabetic nephropathy includes a combination of tight control of blood glucose (goal is $<7 \%$ ), tight control of blood pressure (goal $<130 / 80 \mathrm{mmHg}$ ), lowering of urine albumin, and stopping of smoking (a well-established endothelial cell toxin that also affects progression of kidney disease), the specific blockage of the renin-angiotensin-aldosteron system (RAAS), with either angiotensin-converting enzyme inhibitors (ACEI) or angiotensin II receptor blockers (ARB) and lipid-lowering therapy, especially statins and fibrates.

There is evidence that early therapeutic intervention in patients with chronic kidney disease or diabetes can delay onset of complications and improve outcomes [Atkins, 2010]. For 
example, the UKPDS [Holman, 2008 Bilous, 2008], STENO-2 [Gaede, 2008], and ADVANCE studies [Patel, 2007, Patel, 2008, Zoungas, 2009] demonstrated that tight control of blood glucose level, blood pressure (and lipids in STENO-2) significantly reduced incidence and progression of diabetic kidney disease. In people with type 2 diabetes, inhibition of the renin-angiotensin-aldosterone system using an ACE inhibitor or an ARB decreased the progression from normoalbuminuria to microalbuminuria [Ruggenenti, 2004], reduced the progression from micro albuminuria to macroalbuminuria, [Parving, 2001] and slowed the development of ESRD [Lewis, 2001]. Thus the use of an ACE inhibitor or ARB is now standard therapy for patients with diabetic nephropathy as well as glucose, lipid and blood pressure control. Effective management using evidence-based therapies is the fourth step in tackling diabetic kidney disease.

Like other microvascular complications of diabetes, there are strong associations between glucose control (as measured by hemoglobin A1c [A1C]) and the risk of developing diabetic nephropathy. Patients should be treated to the lowest safe glucose level that can be obtained to prevent or control diabetic nephropathy [Fowler - 2008]. Glycemic control in both type 1 and type 2 diabetes has been associated with reduced appearance of diabetic nephropathy (microalbuminuria) [Hall, 2006]. Control of glycemia in type 2 diabetic patients with nephropathy represents some peculiar aspects. Today there is no longer any doubt that tight glycemic control prevents the onset or progression of diabetic nephropathy in type 2 diabetic patients as it does in type 1 diabetic patients. In the past, it had been thought that once clinically manifest nephropathy had developed, a point of no return was reached beyond which tight glycemic control failed to prevent the further decline in renal function. Nevertheless recent studies from the Steno Hospital showed that glycemic control had some, although less pronounced compared with tight BP control, effect on the rate of progression [Wolf, 2003].

The Diabetes Control and Complications Trial, the United Kingdom Prospective Diabetes Study and the Japanese Diabetes Intervention Study have confirmed that tight glycemic control can prevent the development of all microvascular complications including nephropathy [Looke, 2008].

Once albuminuria occurs, controlling blood pressure assumes critical importance. The use of renin-angiotensin blockade has been a major advance in the management of diabetic nephropathy. Major landmark studies including the Irbesartan Diabetic Nephropathy Trial [Louis, 2001] and RENAAL study [Brenner, 2001] have provided compelling evidence to support the renoprotective effect of angiotensin-II receptor antagonists, independent of blood pressure lowering [Looke, 2008].

The renin-angiotensin system (RAAS) is an important target for hemodynamic disturbances in diabetic kidney disease. Blocking the renin-angiotensin system in diabetes has pluripotent effects on modifying the systemic and glomerular hemodynamic as well as attenuating the pro-inflammatory and pro-fibrotic changes in renal parenchyma [Look, 2008]. The pharmacological agents which block the RAAS slow the progression of renal dysfunction more effectively than other classes of antihypertensive agents. In addition to normalization of systemic and glomerular hypertension, it is now clear that inhibition of the RAAS at various levels have several renoprotective effects, including anti-inflammatory and antifibrotic mechanisms.

Currently available therapies for this blockade are angiotensin converting enzyme inhibitor (ACE-I), angiotensin II type 1 receptor blocker (ARB) and direct renin inhibitor. If the treatment with ACEI and/or ARB does not reach the blood pressure target levels, then they 
can be combined with other drug classes, even before maximizing the dose of each agent. The combination of agents may include calcium channel blockers (especially nondihydropyridine), $\beta$-blockers, diuretics or central $\alpha_{2}$-agonist.

Another concept of a dual blockade of the RAAS has been developed by Mogensen in 2000. Since ACEI and ARB interrupt the RAAS at different levels, the combination of these classes of drugs may have an additive effect on renoprotection. The renoprotective effects of dual blockade of the RAAS with ARB and direct renin inhibitor (aliskiren) may also be effective.

Addition of diuretics has been strongly recommended by KDOQI guidelines to achieve target blood pressures of $<130 \mathrm{mmHg}$ in cases of recalcitrant hypertension in diabetes kidney disease patients. The role for aldosterone antagonist (spironolactone) and statins in prevention of metabolic derangements consequent to RAAS overactivity in DKD is emerging.

The BP-lowering arm of the Action in Diabetes and Vascular disease: preterAx and diamicroN-MR Controlled Evaluation (ADVANCE) study recently reported that the routine administration of a fixed combination of the angiotensin converting enzyme inhibitor perindopril and the diuretic indapamide to a broad cross-section of patients with type 2 diabetes reduced the risk for cardiovascular and kidney outcomes, regardless of initial BP level. More recently, the glucose-lowering arm of ADVANCE reported that intensive glucose lowering based on gliclazide (modified release) reduced the risk for new or worsening nephropathy.

While good glycemic control ameliorates the development of microalbuminuria in type 1 and type 2 diabetes there is increasing evidence that angiotensin-converting enzyme inhibition and angiotensin receptor blockade are ineffective as primary prevention strategies. Blood pressure control remains the mainstay of treating established diabetic renal disease and a multi-factorial intervention approach in those with type 2 diabetes and microalbuminuria appears to be effective in reducing mortality based on data from one study [Walker, 2010].

\section{Aldosterone receptor antagonists}

Experimental studies supporting aldosterone antagonists have shown anti-inflammatory effect, anti-fibrotic properties and suppression of markers of tubular injury, interstitial fibrosis and glomerulosclerosis [Kramer, 2007]. Aldosterone antagonists have generally been considered to have an antiproteinuric effect, and addition of spironolactone to an ACE inhibitor or ARB is associated with a marked and sustained antiproteinuric effect, with the rate of hyperkalemia being similar to placebo. These results are based on studies with small numbers of patients, mostly of very short follow-up. There are no long term data regarding benefit with the combination of ACE inhibitor or ARB and aldosterone antagonists in terms of slowing the progression of diabetic kidney disease. In clinical practice, the use of this combination of agents in patients with low GFR should be undertaken with careful instructions for dietary potassium restriction and avoidance of nonsteroidal antiinflammatory drugs and cyclooxy-genase-2 inhibitors [Satirapoj, 2010].

\section{Peroxisome proliferator-activated receptors (PPAR)-gamma agonists}

Peroxisome proliferator activated receptor- $\gamma$ (PPAR $\gamma$ ) is a ligand activated transcription factor that regulates cell growth, inflammation, lipid metabolism and insulin sensitivity, 
appear to have a role in regulating adipogenesis and blood pressure [Satirapoj, 2010]. PPARY agonists may indirectly suppress the systemic production of a proinflammatory milieu mainly via inhibiting TNF- $\alpha$, PAI-1, and IL-6 expression in adipose tissue. Additionally, PPARY seems to have anti-inflammatory effects on monocytes. PPARY activation can reduce the production of cytokines (TNF-a, IL-1, IL-6), probably through inhibiting the activity of proinflammatory transcription factors such as NF-kB. PPARs have also been implicated in many renal pathophysiological conditions, including diabetic nephropathy and glomerulosclerosis [Ruan, 2008]. Recently it was demonstrated that peroxisome proliferator activated receptor- $\gamma$ (PPAR $\gamma)$ agonists block the deleterious effects of AGEs and exert beneficial actions on diabetic nephropathy. It has been successfully shown that PPAR- $\gamma$ agonists limit high glucose-induced inflammation in proximal tubular cells. PPAR- $\gamma$ agonists also exert anti-fibrotic effects in human proximal tubular cells under high glucose conditions by attenuating the increase in AP-1, TGF- $\beta 1$ and downstream production of the extracellular matrix protein, fibronectin. PPAR- $\gamma$ agonists can improve insulin sensitivity, reduce triglyceride levels and decrease the risk of atherosclerosis. PPARY agonists have been shown to lower blood pressure in animals and humans, perhaps by suppressing the renin-angiotensin (Ang)-aldosterone system (RAAS), including the inhibition of Ang II type 1 receptor expression, Ang-II-mediated signaling pathways, and Ang-II-induced adrenal aldosterone synthesis/secretion [Sugawara, 2011].

\section{Protein restriction}

Animal studies have shown that restriction of dietary protein intake also reduces hyperfiltration and intraglomerular and retards the progression of several models of renal disease, including diabetic glomerulopathy. Reduction of dietary protein has long been proposed as a treatment for CRF and this intervention has shown a modest benefit in slowing loss of filtration function, but adherence to this form of therapy is difficult in diabetes.

\section{Lipid control}

Clinical studies in patients with diabetic nephropathy showed that lipid control can be associated with an additional effect of reduction in proteinuria. Lipid-lowering agents (statins) showed renoprotection in a variety of proteinuric glomerular diseases [Agarwal, 2007]. Although lipid-lowering treatment has been shown to be effective in reducing cardiovascular morbidity and mortality in diabetic patients with hyperlipidemia, their effects on diabetic nephropathy are still unclear due to lack of prospective randomized intervention studies [Chen, 2005]. Early studies have demonstrated that treatment with the HMG CoA reductase inhibitor pravastatin decreases albuminuria in patients with type 2 diabetes [Sasaki, 1990]. Experimental studies demonstrated that lipid-lowering agents exerted a certain degree of renoprotection, through both indirect effects from lipid lowering and a direct effect on cell protection. Therefore, lipid control appears to be important in the prevention and treatment of diabetic nephropathy [Chen, 2005].

\section{Antioxidants}

There have been multiple trials using vitamin $\mathrm{E}$, vitamin $\mathrm{C}$, probucol, taurine, $\mathrm{n}$ acetylcysteine, lipoic acid, and other antioxidant drugs in animals. Animal studies using 
these compounds have been shown to be effective in reducing the development of diabetic kidney disease. It's hard to translate these animal results to humans as many trials in mice and rats are very effective in the particular animal but often ineffective in humans. This may be due to short duration of animal studies, dose differences between animals and humans, and different pathophysiologic processes between animals and humans. Human studies with antioxidants for diabetic nephropathy are limited and have had variable results [Stanton, 2011].

Despite receiving optimal care to control blood pressure and metabolic risk factors as well as inhibition of the renin-angiotensin system in a clinical trial setting, there is a considerable residual risk for cardio-renal complications in patients with diabetic kidney disease. Understanding the molecular milieu of diabetic podocyte injury, an early event in diabetic nephropathy, remains a primary target in identifying novel avenues for early intervention and prevention of severe late complications of this increasingly prevalent disease. Decreasing hyperglycemia's toxic effects, stimulating the endogenous protective factors, controlling obesity and low grade inflammation as well as correction of anemia may represent also areas where novel strategies can be developed and tested to curb this rising global burden of cardio-renal complications in diabetic patients. Correction of anemia improves renal tissue oxygenation, reduces hypoxic damage and associated oxidative stress. There have been several reports suggesting that early erythropoietin treatment may retard the progression of renal diseases and delay the commencement of renal replacement therapy.

Annual screening for microalbuminuria will allow the identification of patients with nephropathy at a point very early in its course. Improving glycemic control, aggressive antihypertensive treatment, and the use of ACE inhibitors or ARBs will slow the rate of progression of nephropathy. In addition, protein restriction and other treatment modalities such as phosphate lowering may have benefits in selected patients.

In conclusion, there are very compelling reasons to better understand the mechanisms underlying diabetic kidney disease, to develop new treatments that can both prevent the development of kidney disease and slow or stop the progression.

\section{References}

[1] ADVANCE Collaborative Group, Patel A, MacMahon S, Chalmers J, Neal B, Woodward M, Billot L, Harrap S, Poulter N, Marre M, Cooper M, Glasziou P, Grobbee DE, Hamet P, Heller S, Liu LS, Mancia G, Mogensen CE, Pan CY, Rodgers A, Williams B: Effects of a fixed combination of perindopril and indapamide on macrovascular and microvascular outcomes in patients with type 2 diabetes mellitus (the ADVANCE trial): A randomised controlled trial. Lancet 370: 829-840, 2007

[2] ADVANCE Collaborative Group, Patel A, MacMahon S, Chalmers J, Neal B, Billot L, Woodward M, Marre M, Cooper M, Glasziou P, Grobbee D, Hamet P, Harrap S, Heller S, Liu L, Mancia G, Mogensen CE, Pan C, Poulter N, Rodgers A, Williams B, Bompoint S, de Galan BE, Joshi R, Travert F: Intensive blood glucose control and vascular outcomes in patients with type 2 diabetes. N Engl J Med 358: 2560- 2572, 2008

[3] Agarwal R. Effects of statins on renal function. Mayo Clin Proc 2007; 82: 1381-90. 
[4] Anjaneyulu M, Chopra K: Effect of irbesartan on the antioxidant defense system and nitric oxide release in diabetic rat kidney. Am J Nephrol 24: 488-496, 2004

[5] Atkins RC, Zimmet P. Diabetic Kidney disease: act now or pay later. Kidney Int. 2010 Mar;77(5):378- 80.

[6] Ayodele OE, Alebiosu CO, Salako BL: Diabetic nephropathy--a review of the natural history, burden, risk factors and treatment. J Natl Med Assoc 96:1445 - 1454, 2004.

[7] B.M. Brenner, M.E. Cooper, D. de Zeeuw, W.F. Keane, W.E. Mitch, H.H. Parving, et al., Effects of losartan on renal and cardiovascular outcomes in patients with type 2 diabetes and nephropathy, N. Engl. J. Med. 345 (2001) 861-869.

[8] Bilous R. Microvascular disease: what does the UKPDS tell us about diabetic nephropathy? Diabet Med, 2008; 25:25-9.

[9] Border WA, Yamamoto T, Noble NA: Transforming growth factor- $\beta$ in diabetic nephropathy. Diabetes Metab Rev 12: 309-339, 1996

[10] Bruijn JA, Roos A, de Geus B, de Heer E: Transforming growth factor-beta and the glomerular extracellular matrix in renal pathology. J Lab Clin Med 123: 34-47, 1994

[11] Burney B, Kalaitzidis R, Bakris G. Novel therapies of diabetic nephropathy. Curr Opin Nephrol Hypertens, 2009; 18:107-11.

[12] Cameron JS: The nephrotic syndrome: A historical review. In: The Nephrotic Syndrome, edited by Cameron JS, Glassock RJ, New York, Marcel Dekker, 1988, pp 3-56

[13] Chiarelli F, Spagnoli A, Basciani F et al (2000) Vascular endothelial growth factor (VEGF) in children, adolescents and young adults with type 1 diabetes mellitus: relation to glycaemic control and microvascular complications. Diabet Med 17:650656

[14] Christensen PK, Larsen S, Horn T, Olsen S, Parving HH: Renal function and structure in albuminuric type 2 diabetic patients without retinopathy. Nephrol Dial Transplant 16:2337-2347, 2001.

[15] Craven P, DeRubertis F (1989) Protein kinase C is activated in glomeruli from streptozotocin diabetic rats. J Clin Invest 83:1667-1675

[16] Deckert T, Poulsen JE: Diabetic nephropathy: Fault or destiny? Diabetologia 21: 178183,1981

[17] Dronavalli S, Duka I, Bakris GL. The pathogenesis of diabetic nephropathy. Nat Clin Pract Endocrinol Metab 2008; 4: 444-452

[18] E.J. Lewis, L.G. Hunsicker, W.R. Clarke, T. Berl, M.A. Pohl, J.B. Lewis, et al., Renoprotective effect of the angiotensinreceptor antagonist irbesartan in patients with nephropathy due to type 2 diabetes, N. Engl. J. Med. 345 (2001) 851-860.

[19] Forbes JM, Cooper ME. Glycation in diabetic nephropathy. Amino Acids 2010 [Epub ahead of print].

[20] Fowler MJ. Microvascular and macrovascular complications of diabetes. Clin Diabetes. 2008;26(2):77-82.

[21] Freedman BI, Bostrom M, Daeihagh P, et al. Genetic factors in diabetic nephropathy. Clin J Am Soc Nephrol 2007;2:1306-1316.

[22] Furuta T, Saito T, Ootaka T, Soma J, Obara K, Abe K, Yoshinaga K: The role of macrophages in diabetic glomerulosclerosis. Am J Kidney Dis 21:480 - 485, 1993.

[23] Gaede P, Lund-Andersen H, Parving HH et al. Effect of a multifactorial intervention on mortality in type 2 diabetes. N Engl J Med, 2008; 358:580-91. 
[24] Gall M-A, Hougaard P, Borch-Johnsen K, et al. Risk factors for development of incipient and overt diabetic nephropathy in patients with noninsulin-dependent diabetes mellitus: prospective observational study. BMJ.1997;314:783-788.

[25] Gerstein H, Mann J, Yi Q, Zinman B, Dinneen S, Hoogwerf B, Halle J, Young J, Rashkow A, Joyce C, Nawaz S, Yusuf S: Albuminuria and risk of cardiovascular events, death, and heart failure in diabetic and nondiabetic individuals. JAMA 286:421-426, 2001

[26] Hall PM. Prevention of pregression in diabetic nephropathy. Diab. Spectr. 2006;19:18-24

[27] Hohenstein B, Hausknecht B, Boehmer K, Riess R, Brekken RA, Hugo CP (2006) Local VEGF activity but not VEGF expression is tightly regulated during diabetic nephropathy in man. Kidney Int 69:1654-1661

[28] Holman RR, Paul SK, Bethel MA et al. 10-year follow-up of intensive glucose control in type 2 diabetes. N Engl J Med, 2008; 359:1577-89.

[29] Hong CY, Chia KS, Ling SL: Urinary protein excretion in type 2 diabetes with complications. J Diabetes Complications 14:259 -265, 2000.

[30] Hovind P, Rossing P, Johnson RJ, Parving HH, Serum Uric Acid as a New Player in the Development of Diabetic Nephropathy, Journal of Renal Nutrition, 21 (1): 124-127, 2011.

[31] Inagaki Y, Yamagishi S, Okamoto T, Takeuchi M, Amano S: Pigment epitheliumderived factor prevents advanced glycation end products-induced monocyte chemoattractant protein-1 production in microvascular endothelial cells by suppressing intracellular reactive oxygen species generation. Diabetologia 2003; 46: 284-287.

[32] Janssen B et al. (2005) Carnosine as a protective factor in diabetic nephropathy: association with a leucine repeat of the carnosinase gene CNDP1. Diabetes 54: 2320-2327

[33] Jones CA, Krolewski AS, Rogus J, Xue JL, Collins A, Warram JH. Epidemic of end-stage renal disease in people with diabetes in the United States population: do we know the cause? Kidney Int 2005;67:1684-1691.

[34] Kanesaki Y, Suzuki D, Uehara G et al (2005) Vascular endothelial growth factor gene expression is correlated with glomerular neovascularization in human diabetic nephropathy. Am J Kidney Dis 45:288-294

[35] Kashihara N, Haruna Y, Kondeti VK, Kanwar YS. Oxidative stress in diabetic nephropathy. Curr Med Chem. 2010;17:4256-4269.

[36] Kim NH, Oh JH, Seo JA et al (2005) Vascular endothelial growth factor (VEGF) and soluble VEGF receptor FLT-1 in diabetic nephropathy. Kidney Int 67:167-177

[37] Klein R, Klein BEK, Moss SE, et al. The 10-year incidence of renal insufficiency in people with type-I diabetes. Diabetes Care. 1999;22:743-751.

[38] Kramer AB, van der Meulen EF, Hamming I, van Goor H, Navis G. Effect of combining ACE inhibition with aldosterone blockade on proteinuria and renal damage in experimental nephrosis. Kidney S240 J Med Assoc Thai Vol. 93 Suppl. 62010 Int 2007; 71: 417-24.

[39] Krishan P, Chakkarwar VA, Diabetic nephropathy: Aggressive involvement of oxidative stress, J Pharm Educ Res Vol. 2, Issue No. 1, June 2011 
[40] Langham RG, Kelly DJ, Gow RM, Zhang Y, Cordonnier DJ, Pinel N, Zaoui P, Gilbert RE: Transforming growth factor-beta in human diabetic nephropathy: Effects of ACE inhibition. Diabetes Care 29: 2670-2675, 2006.

[41] Lee TS, MacGregor LC, Fluharty SJ, King GL (1989) Activation of protein kinase C by elevation of glucose concentration: proposal for a mechanism in the development of diabetic vascular complications. PNAS 86:5141-5145

[42] Levey AS, Bosch JP, Lewis JB, Greene T, Rogers N, Roth D; Modification of Diet in Renal Disease Study Group. A more accurate method to estimate glomerular filtration rate from serum creatinine: a new prediction equation. Ann Intern Med 1999; 130:461-470.

[43] Levey AS, Coresh J, Balk E, Kausz AT, Levin A, Steffes MW, Hogg RJ, Perrone RD, Lau J, Eknoyan G: National Kidney Foundation practice guidelines for chronic kidney disease: evaluation, classification, and stratification. Ann Intern Med 139:137-147, 2003.

[44] Lewis EJ, Hunsicker LG, Clarke WR et al. Renoprotective effect of the angiotensinreceptor antagonist irbesartan in patients with nephropathy due to type 2 diabetes. N Eng J Med, 2001; 345:851-60.

[45] Lin J, Glynn RJ, Rifai N, et al. Inflammation and progressive nephropathy in type 1 diabetes in the diabetes control and complications trial. Diabetes Care 2008;31:2338-43. [PubMed: 18796620]

[46] Liu H, Ren JG, Cooper WL, Hawkins CE, Cowan MR, Tong PY: Identification of the antivasopermeability effect of pigment epithelium- derived factor and its active site. Proc Natl Acad Sci USA 2004; 101: 6605- 6610.

[47] Luk A, Chan JC. Diabetic nephropathy - what are the unmet needs? Diabetes Res Clin Pract. 2008; 82(suppl 1):S15-S20.

[48] Lurbe E, Redon J, Kesani A, Pascual JM, Tacons J, Alvarez V, Batlle D: Increase in nocturnal blood pressure and progression to microalbuminuria in type 1 diabetes. N Engl J Med 347:797-805, 2002.

[49] Lurbe L, Redon J, Kesani A, Pascual JM, Tacons J, Alvarez V, Battle D: Increase in nocturnal blood pressure and progression to microalbuminuria in type 1 diabetes. N Engl J Med 347: 797-805, 2002

[50] Mauer SM, Steffes MW, Brown DM: The kidney in diabetes. Am J Med 70:603-612, 1981.

[51] Mauer SM, Steffes MW, Ellis EN, Sutherland DE, Brown DM, Goetz FC: Structuralfunctional relationships in diabetic nephropathy. J Clin Invest 74: 1143-1155, 1984

[52] Meier M, Menne J, Haller H. Targeting the protein kinase $C$ family in the diabetic kidney: lessons from analysis of mutant mice. Diabetologia 52: 765-775, 2009.

[53] Menne J, Meier M, Park JK, Haller H. Inhibition of protein kinase C in diabetic nephropathy - where do we stand? Nephrol Dial Transplant 2009; 24: 2021-3.

[54] Mogensen CE, Christiansen CK. Blood pressure changes and renal function changes in incipient and overt diabetic nephropathy. Hypertension 1985;7:II64-II73.

[55] Mogensen CE, Poulsen PL (1994) Epidemiology of microalbuminuria in diabetes and in the background population. Curr Opin Nephrol Hypertens 3:248-256 
[56] Mogensen CE. Defintion of diabetic renal disease in insulin dependent diabetes mellitus based on renal function tests. In The Kidney and Hypertension in diabetes mellitus . 3rd ed. Mogensen CE ,ed. London, Kluwer,1996 pp.11

[57] Navarro-Gonzalez JF, Mora-Fernandez C. The role of inflammatory cytokines in diabetic nephropathy. J Am Soc Nephrol 2008; 19: 433-42.

[58] Parving HH, Lehnert H, Jens Bröchner-Mortensen et al. The effect of irbesartan on the development of diabetic nephropathy in patients with type 2 diabetes. $\mathrm{N}$ Engl J Med, 2001; 345:870-8.

[59] Patel A, MacMahon S, Chalmers J et al. and ADVANCE Collaborative Group. Effects of a fixed combination of perindopril and indapamide on macrovascular and microvascular outcomes in patients with type 2 diabetes mellitus (the ADVANCE trial): a randomised controlled trial. Lancet, 2007; 370:829-40.

[60] Patel A, MacMahon S, Chalmers J et al. and ADVANCE Collaborative Group. Intensive blood glucose control and vascular outcomes in patients with type 2 diabetes. $\mathrm{N}$ Engl J Med, 2008; 358:2560-72.

[61] Ravid M, Neumann L, Lishner M. Plasma lipids and the progression of nephropathy in diabetes mellitus type-2: effect of ACE inhibitors. Kidney Int. 1995;47:907910.

[62] Remuzzi G, Bertani T. Pathophysiology of progressive nephropathies. N Engl J Med 1998;12:1448-1456.

[63] Ruan, X., Zheng, F. and Guan, Y. (2008) PPARs and the kidney in metabolic syndrome. Am. J. Physiol. Renal Physiol. 294, F1032-F1047

[64] Ruggenenti P, Fassi A, Ilieva AP et al. Preventing microalbuminuria in type 2 diabetes. N Engl J Med., 2004; 351(19):1941-51.

[65] Ruggenenti P, Gambara V, Perna A, Bertani T, Remuzzi G: The nephropathy of noninsulin-dependent diabetes: Predictors of outcome relative to diverse patterns of renal injury. J Am Soc Nephrol 9: 2336-2343, 1998

[66] Ruggenenti P, Remuzzi G. Nephropathy of type 2 diabetes mellitus. J Am Soc Nephrol1998; 9: 2157-2169

[67] Ruggenenti P, Remuzzi G. Time to abandon microalbuminuria? Kidney Int. 2006;70:1214-22.

[68] Ruggenenti P, Remuzzi G: Nephropathy of type 1 and type 2 diabetes: diverse pathophysiology, same treatment? Nephrol Dial Transplant 15:1900-1902, 2000

[69] Sasaki T, Kurata H, Nomura K, et al: Amelioration of proteinuria with pravastatin in hypercholesterolemic patients with diabetes mellitus. Jpn J Med 29:156-163, 1990

[70] Satchell SC, Tasman CH, Singh A et al (2006) Conditionally immortalized human glomerular endothelial cells expressing fenestrations in response to VEGF. Kidney Int 69:1633-1640

[71] Satchell SC, Tooke JE. What is the mechanism of microalbuminuria in diabetes: a role for the glomerular endothelium? Diabetologia 51: 714-725, 2008

[72] Satirapoj B. Review on pathophysiology and treatment of diabetic kidney disease. J Med Assoc Thai. 2010;93 (suppl 6):S228 -S241.

[73] Schalkwijk CG, Poland DC, van Dijk W et al (1999) Plasma concentration of C-reactive protein is increased in type I diabetic patients without clinical macroangiopathy and correlates with markers of endothelial dysfunction: evidence for chronic inflammation. Diabetologia 42:351-357 
[74] Schmid H, Boucherot A, Yasuda Y, Henger A, Brunner B, Eichinger F, Nitsche A, Kiss E, Bleich M, Grone HJ, Nelson PJ, Schlondorff D, Cohen CD, Kretzler M: Modular activation of nuclear factor-kappaB transcriptional programs in human diabetic nephropathy. Diabetes 55: 2993-3003, 2006

[75] Schrier RW, Estacio RO, Esler A, Mehler P: Effects of aggressive blood pressure control in normotensive type 2 diabetic patients on albuminuria, retinopathy and strokes. Kidney Int 61:1086-1097, 2002.

[76] Song Y, Manson JE, Buring JE, Liu S. A prospective study of red meat consumption and type 2 diabetes in middle-aged and elderly women: the Women's Health Study. Diabetes Care. 2004;27:2108-2115.

[77] Srivastava SK, Ramana KV, Bhatnagar A. Role of aldose reductase and oxidative damage in diabetes and the consequent potential for therapeutic options. Endocr Rev. 2005;26:380 -392.

[78] Stanton RC Oxidative stress and diabetic kidney disease. Curr Diab Rep 2011 Aug;11(4):330-6.

[79] Stehouwer CD, Gall MA, Twisk JW, Knudsen E, Emeis JJ, Parving HH (2002) Increased urinary albumin excretion, endothelial dysfunction, and chronic low-grade inflammation in type 2 diabetes: progressive, interrelated, and independently associated with risk of death. Diabetes 51:1157-1165

[80] Sugawara A, Uruno A, Kudo M, Matsuda K, Yang CW, Ito S. Sugawara A, Uruno A, Kudo M, Matsuda K, Yang CW, Ito S., Korean J Intern Med 26:19 (2011)

[81] The Diabetes Control and Complications Trial Research Group, The effect of intensive treatment of diabetes on the development and progression of longterm complications in insulin-dependent diabetes mellitus, N. Engl. J. Med. 329 (1993) 977-986.

[82] The United States Renal Data System. http://www.usrds.org/ Annual Data Report 2010

[83] Thomas M.C. Anemia in diabetes: marker or mediator of microvascular disease? Nat. Clin. Pract. Nephrol. 2007;3:20-30.

[84] Thomas MC, Cooper ME, Rossing K, Parving HH: Anaemia in diabetes: is there a rationale to TREAT? Diabetologia 49: 1151-1157, 2006.

[85] Tilton RG et al. Prevention of hemodynamic and vascular filtration changes in diabetic rats by aldose reductase inhibitors. Diabetes 1989; 38: 1258-1270.

[86] United Kingdom Prospective Diabetes Study (UKPDS) Group, Intensive blood-glucose control with sulphonylureas or insulin compared with conventional treatment and risk of complications in patients with type 2 diabetes, Lancet 352 (1998) 837-853.

[87] Walker JD, An update on diabetic renal disease, Br J Diabetes Vasc Dis 2010;10:219223.

[88] Warram JH, Gearin G, Laffel L, Krolewski AS (1996) Effect of duration of type I diabetes on the prevalence of stages of diabetic nephropathy defined by urinary albumin/creatinine ratio. J Am Soc Nephrol 7:930-937

[89] Y. Ohkubo, H. Kishikawa, E. Araki, T. Miyata, S. Isami, S. Motoyoshi, et al., Intensive insulin therapy prevents the progression of diabetic microvascular complications in Japanese patients with non-insulin dependent diabetes mellitus: a randomized prospective 6-year study, Diabetes Res. Clin. Prac. 28 (1995) 103-117. 
[90] Zoungas S, de Galan BE, Ninomiya T et al. The combined effects of routine blood pressure lowering and intensive glucose control on macrovascular and microvascular outcomes in patients with type 2 diabetes; new results from ADVANCE. Diabetes Care, 2009; 32:2068-74 


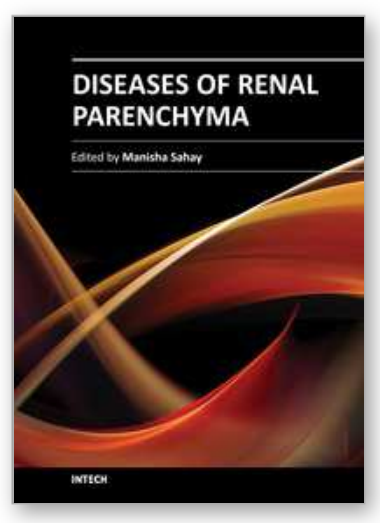

\author{
Diseases of Renal Parenchyma \\ Edited by Prof. Manisha Sahay
}

ISBN 978-953-51-0245-8

Hard cover, 304 pages

Publisher InTech

Published online 16, March, 2012

Published in print edition March, 2012

Clinical nephrology is an evolving speciality in which the amount of information is growing daily. This book gives quick access to some important clinical conditions encountered in nephrology including the diseases of glomeruli, tubules and interstitium. It presents the latest information on pathophysiology, diagnosis and management of important diseases of renal parenchyma. The information is presented in a very user friendly and accessible manner while the treatment algorithms enable the reader to quickly access expert advice on arriving at the most appropriate treatment regimen. The book discusses the renal involvement in various systemic diseases including diabetes and autoimmune diseases. Diabetic nephropathy is fast becoming the commonest cause of end stage renal disease all over the globe and is discussed in this book. The editors believe that this book will be a valuable addition to the reader's library.

\title{
How to reference
}

In order to correctly reference this scholarly work, feel free to copy and paste the following:

Rodica Mihăescu, Corina Şerban, Simona Drăgan, Romulus Timar, loana Mozoş, Marius Craina and Adalbert Schiller (2012). Diabetes and Renal Disease, Diseases of Renal Parenchyma, Prof. Manisha Sahay (Ed.), ISBN: 978-953-51-0245-8, InTech, Available from: http://www.intechopen.com/books/diseases-of-renalparenchyma/diabetes-and-renal-diseases

\section{INTECH}

open science | open minds

\author{
InTech Europe \\ University Campus STeP Ri \\ Slavka Krautzeka 83/A \\ 51000 Rijeka, Croatia \\ Phone: +385 (51) 770447 \\ Fax: +385 (51) 686166 \\ www.intechopen.com
}

\author{
InTech China \\ Unit 405, Office Block, Hotel Equatorial Shanghai \\ No.65, Yan An Road (West), Shanghai, 200040, China \\ 中国上海市延安西路65号上海国际贵都大饭店办公楼 405 单元 \\ Phone: +86-21-62489820 \\ Fax: +86-21-62489821
}


(C) 2012 The Author(s). Licensee IntechOpen. This is an open access article distributed under the terms of the Creative Commons Attribution 3.0 License, which permits unrestricted use, distribution, and reproduction in any medium, provided the original work is properly cited. 